(с) Е.В. Мишарина*, А.В. Тиселько, М.И. Ярмолинская., И.Ю. Коган, Е.И. Абашова, Н.В. Боровик

Научно-исследовательский институт акушерства, гинекологии и репродуктологии имени Д.О. Отта, Санкт-Петербург

У больных сахарным диабетом 1 типа (СД1) имеют место ановуляция, непроходимость маточных труб, мужской фактор бесплодия и другие причины бесплодия, требующие проведения процедуры экстракорпорального оплодотворения (ЭКО).

Представлено описание 20 клинических случаев родивших пациенток с СД1, беременность у которых наступила в результате вспомогательных репродуктивных технологий (ВРТ). Одной из пациенток ЭКО выполнено дважды. Средний возраст пациенток, обратившихся в отделения ВРТ, составил $33 \pm 6$ года, длительность бесплодия составила от 3 до 15 лет. У двух пациенток (с синдромом Шерешевского-Тернера и синдромом Свайера) были использованы ооциты донора. У 70\% пациенток использовался метод постоянной подкожной инфузии инсулина (ППИИ). Оценку углеводного обмена проводили измерением уровня гликированного гемоглобина $\mathrm{A}_{1 \mathrm{c}}\left(\mathrm{Hb}_{1 \mathrm{c}}\right)$, уровня глюкозы в плазме не менее 8 раз в сутки, по результатам непрерывного мониторирования глюкозы.

В результате проведения ЭКО в 18 случаях наступила одноплодная беременность, в трех случаях - многоплодная (дихориальная диамниотическая двойня). В сроке 37-39 нед родоразрешены 12 беременных, в сроки 34-36 нед 9 беременных. Во всех случаях беременность закончилась рождением живых здоровых детей. Вес выше 90-го процентиля имели 6 новорожденных.

КЛЮЧЕВЫЕ СЛОВА: сахарный диабет 1 типа; беременность; ВРТ; ЭКО; постоянная подкожная инфузия инсулина; клинический случай

\title{
IN VITRO FERTILIZATION AS A METHOD OF INFERTILITY TREATMENT IN WOMEN WITH TYPE 1 DIABETES MELLITUS
}

(c) Elena V. Misharina*, Alena V. Tiselko, Maria I. Yarmolinskay, Igor Y. Kogan, Elena I. Abashova, Natalia V. Borovik

Some patients with type 1 diabetes have anovulation, tubal occlusion, male factor and other causes of infertility which require IVF procedure.

We examined 20 women with type 1 diabetes, in which pregnancy occurred as a result of ART, at the stage IVF protocol planning, during pregnancy course and delivery. One of the patients underwent IVF procedure twice. Mean age of the patients who applied to the Department of assisted reproductive technologies was $33 \pm 6$ years, duration of infertility varied from 3 to 15 years. Two patients were performed donor oocytes transfer (patients with Turner Syndrome and Swyer Syndrome). In 70\% of patients the method of CSII was used. Assessment of carbohydrate metabolism was based on the level of glycated hemoglobin $\mathrm{A}_{1 \mathrm{c}}\left(\mathrm{HbA}_{1 c}\right)$, plasma glucose level measured at least 8 times a day and results of continuous glucose monitoring (CGM).

18 singleton pregnancies and three multiple gestations (dichorionic diamniotic twins) occurred as a result of IVF. In 12 women delivery occurred on the 37-39th weeks of gestation, in 9 patients pregnancy was terminated on the 34-36th weeks. In all the cases it was live birth. Birth weight above the 90th percentile had 6 newborns.

KEYWORDS: diabetes mellitus type 1; pregnancy; assisted reproduction; IVF; continuous subcutaneous infusion of insulin; case report

Сахарный диабет 1 типа (СД1) оказывает неблагоприятное влияние на репродуктивную систему женщины. Частота нарушений менструального цикла колеблется от 29,7 до 74,9\% у женщин с СД1 [1-3]. В своем исследовании A. Daltsidou и соавт. (2010) показали, что при СД1 нарушения менструального цикла встречаются в 7,8 раза чаще, чем у женщин без СД [4]. Гормональная недостаточность функции яичников у больных СД1 обусловлена усилением опиоидного и дофаминергического торможения продукции гонадотропин-рилизинг-гормона гипоталамусом и повреждением механизма положительной обратной связи между яичниками и гипофизом [2].
По эпидемиологическим данным, каждая шестая пара в мире нуждается в лечении по поводу отсутствия желаемой беременности. По данным одних исследователей, доля больных СД1, предъявляющих жалобы на бесплодие, составляет около 17\% и не превышает популяционный уровень [5]. В другом исследовании Whitworth K.W. и соавт. отмечают, что вероятность наступления беременности в одном менструальном цикле у больных СД1 по сравнению с популяционными данными существенно ниже (на 24\%) этого показателя вне зависимости от наличия нарушений менструального цикла [6]. Улучшение качества лечения СД1, введение в практику интенсивной 
инсулинотерапии, аналогов инсулина ультракороткого и продленного действия и адекватного контроля гликемии в последние два десятилетия оказали положительное действие на показатели фертильности у больных СД1. Однако у части больных СД1 имеют место ановуляция, непроходимость маточных труб, мужской фактор бесплодия и другие причины бесплодия, требующие стимуляции овуляции и/или экстракорпорального оплодотворения (ЭКО). На данный момент в развитых странах число детей, рожденных после ЭКО, составляет от 1 до 3\% от общего числа родившихся [7]. В литературе описаны единичные случаи использования вспомогательных репродуктивных технологий (ВРТ) у больных СД1 - один, два и девять циклов ЭКО соответственно [8-10]. Авторы отмечают, что по основным показателям эффективности ЭКО у женщин с хорошей компенсацией СД (по показателям $\mathrm{HbA}_{1 c}$ ) значимых отличий в количестве и качестве ооцитов, а также количестве перенесенных и криоконсервированных эмбрионов хорошего качества выявлено не было. Это же касалось частоты наступления клинической беременности [11]. Все вышесказанное делает данную группу пациенток возможными участницами программ ВРТ при соблюдении ряда условий.

При подготовке к проведению лечения бесплодия у больных СД1 основной задачей является достижение целевых показателей уровня глюкозы в крови, для чего используются интенсивная инсулинотерапия с помощью режима множественных инъекций инсулина (МИИ) или постоянной подкожной инфузии инсулина (ППИИ) [12-15].

\section{ОПИСАНИЕ СЛУЧАЕВ}

В нашей клинике за десять лет акушерами-гинекологами и эндокринологами совместно наблюдались 20 женщин с СД1, беременность у которых наступила в результате ЭКО. У одной из пациенток ЭКО выполнялось дважды (с интервалом 3 года).

Средний возраст пациенток, обратившихся в отделения ВРТ, составил $33 \pm 6$ года (от 27 до 44 лет). ИМТ был

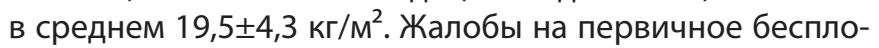
дие предъявили 12 больных, вторичное - 8 больных. Длительность бесплодия у обратившихся женщин составила от 3 до 15 лет. Причины отсутствия беременности были различными. У 5 пациенток при лапароскопии был выявлен наружный генитальный эндометриоз I и II степеней, проведена электрокоагуляция очагов эндометриоза с последующей терапией агонистами гонадотропинов в течение 6 циклов. Ановуляция была у 4 пациенток (у трех связана с наличием синдрома поликистозных яичников и у одной - с гиперпролактинемией, уровень пролактина был скорректирован каберголином). Проводившаяся стимуляция овуляции антиэстрогенами и впоследствии рекомбинантным фолликулостимулирующим гормоном (ФСГ) не имела эффекта. В семи случаях причиной бесплодия стал трубный фактор. В двух случаях причиной бесплодия был мужской фактор. Две пациентки имели хромосомные нарушения.

Среди наиболее часто встречаемых сопутствующих заболеваний были аутоиммунный тиреоидит, субклинический гипотиреоз (у 40\% женщин), компенсированный приемом тиреоидных препаратов и хронический пие- лонефрит (40\% женщин). У двух пациенток был выявлен антифосфолипидный синдром.

Длительность заболевания диабетом составила от одного года до 24 лет (в среднем 12,7士5, 1 года). Осложнения сахарного диабета имели 15 пациенток, направленных на ЭКО: непролиферативную ретинопатию - 13 женщин, диабетическую нефропатию в стадии микроальбуминурии (ДН, ХБП С1 (2) А2) - две пациентки и диабетическую полинейропатию - три пациентки.

Результаты физикального, лабораторного

и инструментального исследования

Перед вступлением в протокол ЭКО 12 пациенток были переведены на постоянную подкожную инфузию инсулина, 8 больных получали МИИ. В сроке 7-12 нед еще 3 пациентки были переведены на ППИИ. Контроль углеводного обмена проводили по $\mathrm{HbA}_{1 c^{\prime}}$ уровню глюкозы в плазме не менее 8 раз в сутки; результатам непрерывного мониторирования глюкозы. За 3 мес до вступления в протокол ЭКО все пациентки имели близкий к физиологическому $\mathrm{HbA}_{1 c}$ (в среднем составил 6,2 $20,5 \%$ ).

Целевой уровень глюкозы был определен как 3,5-7,8 ммоль/л (натощак 3,5-5,3 ммоль/л, постпрандиальный через 1 ч - 7,8 ммоль/л, через 2 ч - 6,7 ммоль/л). Все женщины получали аналоги инсулина ультракороткого действия для болюсного введения (аспарт, лизпро); группа женщин, использующих МИИ, получали аналоги инсулина продленного действия (детемир, гларгин). Длительность непрерывного мониторирования глюкозы (НМГ) у беременных составила от 288 до 432 ч. При использовании ППИИ удалось достичь целевых значений гликемии в 74\% (45-85) времени суток, что было значимо $(p<0,01)$ выше этого показателя в группе женщин, использовавших МИИ 57\% (32-62) времени суток.

\section{Лечение}

Большей части пациенток (18) была проведена контролируемая стимуляция суперовуляции рекомбинантным ФСГ в программе ЭКО и ЭКО с интраплазматической инъекцией сперматозоидов (ЭКО ИКСИ) в протоколе с применением антагонистов гонадотропин-рилизинг-гормона. Введение рекомбинантных гонадотропинов проводилось ежедневно со 2-3-го дня менструального цикла в течение 7-9 дней, суммарная доза гонадотропинов составила 1300-1400 МЕ. Критерием назначения триггера финального созревания ооцитов являлось достижение тремя лидирующими фолликулами среднего размера не менее 17 мм. В качестве триггера финального созревания ооцитов использовались препараты хорионического гонадотропина человека (чХГ). Через 36 ч после введения триггера овуляции проводилась трансвагинальная пункция фолликулов и аспирация ооцитов под внутривенной анестезией. При трансвагинальной пункции аспирировано от 3 до 10 ооцитов. Оплодотворение проводилось стандартным методом (в 9 случаях) или методом ИКСИ (в 9 случаях). Оценку оплодотворения проводили через 17 ч. Перенос одного эмбриона выполнен у 12 пациенток, двух эмбрионов у 7 пациенток на 5-й день развития (качество морулы Gr 4, бластоцисты ЗАА, ЗАВ). Качество эмбрионов оценивалось согласно морфологическим критериям оценки, предложенным D. Gardner, W. Schoolcraft [16]. 
У двух пациенток имелись абсолютные показания для использования ооцитов донора в связи с наличием у них дисгенезии гонад. Одна пациентка имела синдром Шерешевского-Тернера (кариотип 45Х0, диагноз СД1 подтверждён на основании базального уровня С-пептидов 0,18 нг/мл), другая - синдром Свайера (кариотип 46,XY, диагноз СД1 установлен на основании клинической картины: дебют в 23 года, уровень гликемии в дебюте 25 ммоль/л, кетоацидоз тяжелой степени,

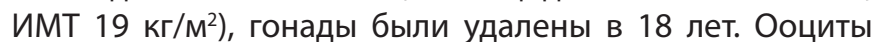
женщин-доноров были оплодотворены спермой мужей женщин-реципиентов. В протоколе с применением заместительной гормональной терапии препаратами эстрогенов и прогестерона обеим пациенткам был совершен перенос одного эмбриона на стадии бластоцисты ЗАА.

Пациенткам с антифосфолипидным синдромом (АФС) в протоколе ЭКО вводились иммуноглобулины, проводилась терапия антикоагулянтами.

Все пациентки находились под наблюдением эндокринолога в связи с неизбежными колебаниями суточной потребности в инсулине в ходе стимуляции суперовуляции за счет дополнительной гормональной нагрузки. Со дня введения чХГ (человеческого хорионического гонадотропина) пациенткам был назначен прогестерон интравагинально. В одном из исследований [17] показано, что микронизированный прогестерон не изменяет значения гликемии в течение суток и $\mathrm{HbA}_{1 c}$. Суточная доза инсулина в протоколах ЭКО увеличивалась на 15-25\%, особенностью явилось увеличение болюсных доз инсулина в вечерние часы, что является характерным для пациенток с СД1 и СД2, получающих препараты прогестерона. Болюсные дозы инсулина были увеличены на 15-20\% на завтрак и обед; на ужин - на 20-25\%. Доза базального инсулина также возросла на 20-25\% у больных, находящихся на МИИ, и у пациенток, использовавших ППИИ (увеличена с 00 до 03 ч на 25\%, с 03 до 09 ч на 25-30\%).

\section{Исход и результаты последующего наблюдения}

Беременность наступила с первой попытки у 11 женщин. У трех пациенток беременность наступила после переноса криоконсервированных эмбрионов со второй попытки, у двух - с третьей попытки. Двум пациенткам было проведено дополнительно 2 и 3 контролируемых стимуляции суперовуляции соответственно. У обеих беременность наступила после переноса криоконсервированных эмбрионов. У женщины с синдромом Шерешевского-Тернера беременность наступила после третьего переноса эмбриона, у больной с синдромом Свайера - после первого переноса эмбриона. Через 3 года пациентке с синдромом Свайера был повторно выполнен перенос одного криоконсервированного эмбриона на стадии бластоцисты (ЗАА). Со дня введения чХГ донору женщинам-реципиентам совместно с эстрогенами был назначен микронизированный прогестерон интравагинально.

В результате проведения ЭКО в 18 случаях наступила одноплодная беременность, в трех случаях - многоплодная (дихориальная диамниотическая двойня). В ранние сроки беременности все пациентки получали сохраняющую терапию в заданных режимах. С 12-14-й недели было начато постепенное снижение доз вводимых гор- монов, с последующей отменой к 16 нед. У пациенток с СД1 после ЭКО не отмечалось достоверного снижения потребности в инсулине, характерного для I триместра беременности. После 16 нед беременности динамика потребности в инсулине, базис-болюсное соотношение доз инсулина не отличалось от показателей пациенток с СД1, у которых беременность наступила самопроизвольно.

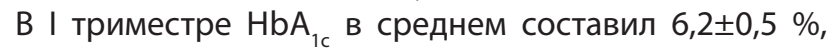
что не отличалось от уровня, с которым пациентки вступали в протокол ЭКО. НbА

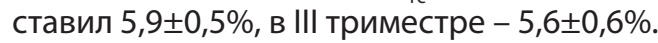

Всем пациенткам во II и III триместрах проводилась профилактика преэклампсии и плацентарной недостаточности, так как беременность, отягощенная СД1, характеризуется повышенной частотой развития гипертензивных нарушений и случаев преэклампсии (15-20\% против 5-7\%) [18]. В нашей группе выраженные гипертензивные нарушения имели место у двух пациенток, у обеих длительность СД1 составила более 10 лет, обе имели диабетическую нефропатию: ХБП С1(2) А2. Во время беременности показатели общих анализов мочи у всех женщин были в пределах референсных значений. Скорость клубочковой фильтрации составила $106,14 \pm 22,11$ мл/мин/1,73м², суточная потеря белка не превышала 0,01-0,08 г, лишь у одной пациентки этот показатель составил 0,29 г. Следует отметить, что одна из пациенток во время первой беременности получала инсулинотерапию в режиме МИИ, перед второй беременностью была переведена на ППИИ. Во время первой беременности у нее развилась преэклампсия тяжелой степени, во вторую - гипертензивные нарушения были менее выраженными. Снижение частоты и тяжести гипогликемических состояний на фоне применения систем НМГ и ППИИ уменьшает частоту осложнений беременности СД1, играет позитивную роль в снижении частоты преэклампсии по сравнению с частотой данного осложнения у больных, использующих режим МИИ [19].

Пациенткам с АФС в I и во II триместрах беременности было проведено 3 курса иммуноглобулинов, а также продолжено применение антикоагулянтов, которое было начато до вступления в протокол ЭКО под контролем свертывающих показателей крови. У 4 пациенток была выявлена анемия легкой степени тяжести, у одной - средней степени тяжести, отмечено снижение сывороточного железа; всем был рекомендован прием препаратов железа.

При выполнении ультразвукового и биохимического скрининга в I триместре, ультразвукового скрининга во II триместре пороков развития выявлено не было. При ультразвуковом исследовании в III триместре были выявлены признаки диабетической фетопатии у двух плодов: утолщение подкожной зоны до 5 мм с типичным двойным контуром вокруг головки плода, увеличение толщины межжелудочковой перегородки более 4,5 мм, отмечена тенденция к развитию крупного плода. Допплерометрические показатели маточно- и плодово-плацентарного кровотока были в пределах референсных значений в течение всего периода наблюдения в 19 случаях. Исследование проводилась 2 раза в неделю после 32-34 нед беременности. В двух случаях при сроке беременности 34-35 нед отмечено повышение резистентности кровотоку в артерии пуповины без централи- 
зации плодового кровотока - нарушение гемодинамики І степени.

Общая прибавка массы тела за беременность в среднем составила 11,8ะ4,1 кг (от 7 до 16 кг).

Всем беременным было проведено родоразрешение путем операции кесарева сечения в условиях спинальной анестезии. В сроки 37-39 нед родоразрешено 12 беременных, в сроки 34-36 недель - 9 беременных. Три пациентки, беременные двойней, также были родоразрешены досрочно: при сроке 34 нед (вес плодов 2440 г и 2070 г), при сроке 35 нед (вес плодов 2180 г и 2080 г) и при сроке 36 нед (вес плодов 2380 г и 2620 г). Причиной досрочного родоразрешения послужило преждевременное излитие вод у семи пациенток, развитие родовой деятельности у пациентки с полным предлежанием плаценты, нарушение жизнедеятельности плода по данным кардиотокографии (снижение количества шевелений у плода) у одной пациентки.

Для оценки веса новорожденных использовались принятые в РФ перцентильные шкалы, разработанные Г.М. Дементьевой [20]. У 18 новорожденных вес при рождении соответствовал сроку гестации и 25-89 перцентилям. Вес выше 90-го перцентиля имели 6 новорожденных. Оценка по шкале Апгар у детей с доношенным сроком составила 8 баллов, в случаях преждевременных родов - 7-8 баллов. Целевая гликемия в родах составляла 4,5-7,5 ммоль/л (в плазме крови) [21]. При извлечении уровень глюкозы у матерей составил 6,6士1,0 ммоль/л (5,2-7,5 ммоль/л), у плодов 3,1 $\pm 0,5$ ммоль/л (2,2-3,4 ммоль/л). Гипогликемия новорожденного в родах была отмечена в 1 случае и составила 2,0 ммоль/л при рождении. Неонатальная гипогликемия (через 2 ч после родов) была зафиксирована у 4 детей $1,6 \pm 0,2$ ммоль/л.

В послеродовом периоде осложнений не наблюдалось. Следует отметить, что в раннем послеродовом периоде (до 3 сут) при физиологической лактации потребность в инсулине снижалась при каждом кормлении на 20-30\% в течение дня, в ночные часы - до 50\% [12, 14, $22,23]$. Матери и дети были выписаны домой на 8-9-е сутки. На второй этап выхаживания были переведены две двойни, рожденные при сроках 34 и 35 нед.

Во всех случаях беременности у женщин с СД1, наступившие после применения ЭКО, закончились рождением живых здоровых детей.

\section{ОБСУЖДЕНИЕ}

Достижение физиологических значений гликемии у больных с СД1 является основным условием благополучного течения и исходов беременности. Улучшение качества лечения СД1, введение в практику ИИТ (интенсивной инсулинотерапии), ППИИ и адекватного контроля гликемии в последние два десятилетия, несомненно, оказало положительное действие на показатели фертильности и исходы течения беременности и родов. В литературе имеются единичные сообщения об эффективности применения методов ВРТ у пациенток с СД1, крупные клинические исследования по этой проблеме отсутствуют. Все сообщения сделаны с привлечением небольшой когорты больных СД1. Однако в одном из клинических исследований было сделано сравне- ние основных показателей результативности проведения программ ЭКО у больных СД1 и без него. Показано, что по основным показателям эффективности проведения ЭКО у женщин с хорошей компенсацией СД по данным $\mathrm{HbA}_{1 с}$ достоверных отличий в количестве и качестве ооцитов, а также количестве перенесенных и криоконсервированных эмбрионов хорошего качества выявлено не было. Это же касалось частоты наступления клинической беременности. Надо отметить, что за 3 мес до вступления в протокол все больные СД1 были переведены на ИИТ с тщательным гликемическим контролем [11]. У пациенток, имевших нормальные параметры овариального резерва, дозы гонадотропинов для стимуляции овуляции были несколько ниже, чем у 5 пациенток с наружным генитальным эндометриозом, и не отличались от доз гонадотропинов для стимуляции овуляции у пациенток без СД, так же как и частота наступления клинической беременности.

Эксперты в области лечения СД во время беременности в настоящее время выбирают метод постоянной подкожной инфузии инсулина с помощью инсулиновой помпы [15]. Авторы подчеркнули, что условием достижения компенсации углеводного обмена являются формирование у данных пациенток мотивации к достижению «жесткого» гликемического контроля и обучение навыкам помповой инсулинотерапии на этапе планирования беременности.

Материнская гипергликемия приводит к повышенному уровню глюкозы и инсулина в крови плода. Многочисленные исследования подтвердили связь между повышением плазменного уровня глюкозы и частотой врожденных пороков развития плода (ВПР). Риск ВПР у пациенток с СД может быть в 3-4 раза выше популяционного и имеет прямую корреляцию с $\mathrm{HbA}_{1 c}$ в крови. Этот риск снижается по мере приближения уровня $\mathrm{HbA}_{1 с}$ к физиологическим значениям (менее 5,8\%).

Применение помповой инсулинотерапии и метода НМГ у женщин с СД1 в центре «Новые технологии в лечении сахарного диабета» на базе ФГБНУ «НИИ АГиР им. Д.О. Отта» привело к снижению числа случаев перинатальной смертности, уменьшению частоты тяжелой преэклампсии с 38 до 9,6\%, увеличению срока родо-

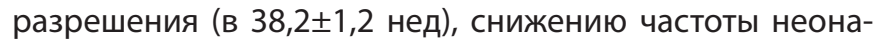
тальной гипогликемии с 31 до 14\%. По данным нашего центра, макросомия выявляется у 58\% детей от матерей с незапланированной беременностью и у $23 \%$ детей от матерей, планировавших беременность. В нашей группе вес выше 90 перцентиля имели 6 новорожденных (25\%), неонатальную гипогликемию - 4 новорожденных (15\%). Тяжелая преэклампсия имела место у 2 пациенток (10\%). Срок родоразрешения составил 38 нед. Наиболее эффективным и безопасным, как показывает международный и наш клинический опыт, является перевод пациенток с СД1 на помповую инсулинотерапию на этапе планирования беременности. Представленные данные позволяют рекомендовать методы ППИИ и НМГ как безопасные и эффективные в лечении больных СД1 во время беременности, родов и в послеродовом периоде.

Результаты применения методов ВРТ у пациенток с СД1 и сопутствующей эндокринной патологией в проведенном нами исследовании подтвердили данные литературы о том, что частота наступления беременности в данной 
группе больных не отличается от частоты наступления беременности у пациенток без СД. Частота невынашивания беременности в группе больных СД1 не превышала соответствующие показатели у женщин без СД.

Использование ППИИ и адекватного контроля гликемии привело к достижению целевых показателей углеводного обмена в периоды контролируемой стимуляции овуляции и во время беременности, что согласуется с данными ряда авторов [12, 15, 16], которые подтверждают эффективность и безопасность использования данного метода лечения у больных с СД1. Частота перинатальных осложнений в проведенном исследовании не превышала эти показатели у больных без применения ВРТ.

\section{ЗАКЛЮЧЕНИЕ}

Проведение ВРТ у больных СД1 является возможным. Основными условиями для выполнения ЭКО у данной группы пациенток являются: достижение физиологических показателей гликемии до протокола ЭКО и на этапе всей беременности, своевременная и адекватная оценка наличия микрососудистых и макрососудистых осложнений.

Использование у женщин с СД1 современных методов лечения, таких как ППИИ, применение аналогов инсулина ультракороткого действия, делает метод ВРТ эффективным и безопасным и позволяет женщинам с бесплодием успешно выносить беременность и родить здоровых детей.

\section{ДОПОЛНИТЕЛЬНАЯ ИНФОРМАЦИЯ}

Согласие пациентов. При поступлении в стационар все пациентки дали согласие на обработку персональных данных. Все наши пациентки дали информированное согласие на публикацию медицинских данных, представленных в статье (в обезличенной форме) в журнале «Сахарный диабет».

Конфликт интересов. Авторы декларируют отсутствие явных и потенциальных конфликтов интересов, связанных с публикацией настоящей статьи.

Все авторы внесли существенный вклад в проведение исследования и подготовку статьи, прочли и одобрили финальную версию перед публикацией.

\section{СПИСОК ЛИТЕРАТУРЫ | REFERENCES}

1. Айламазян Э.К., Потин В.В., Тарасова М.А., и др. Гинекология от пубертата до постменопаузы. - М.: МЕДпресс-информ; 2004. [Aylamazyan EK, Potin W, Tarasova MA, et al. Ginekologiya ot pubertata do postmenopauzy. Moscow: MEDpress-inform; 2004. (In Russ.)]

2. Толпыгина М.Г., Абашова Е.И., Боровик Н.В., Тиселько А.В. Патогенез нарушения функции яичников у женщин с сахарным диабетом 1-го типа // Журнал акушерства и женских болезней. 2018. — T. 67. — №1. - C. 5-12. [Tolpigina MG, Abashova El, Borovik $\mathrm{NV}$, Tiselko AV. Diabetes mellitus and female reproductive system. Journal of Obstetrics and Women's Diseases. 2018;67(1):5-12. (In Russ.)] doi: 10.17816/JOWD6715-12

3. Толпыгина М.Г., Потин В.В., Тарасова М.А. Функция яичников у женщин с сахарным диабетом 1-го типа // Журнал акушерства иженских болезней. - 2014. - Т. 63. — №3. — C. 53-55. [Tolpygina MG, Potin W, Tarasova MA. Ovarian function in women with type 1 diabetes mellitus. Journal of Obstetrics and Women's Diseases. 2014;63(3):53-55. (In Russ.)] doi: 10.17816/JOWD63353-57

4. Deltsidou A, Lemonidou C, Zarikas V, et al. Oligomenorrhoea in adolescents with type 1 diabetes mellitus: relationship to glycaemic control. Eur J Obstet Gynecol Reprod Biol. 2010;153(1):62-66. doi: 10.1016/j.ejogrb.2010.07.027

5. Strotmeyer ES, Steenkiste AR, Foley TP, et al. Menstrual Cycle Differences Between Women With Type 1 Diabetes and Women Without Diabetes. Diabetes Care. 2003;26(4):1016-1021. doi: 10.2337/diacare.26.4.1016

6. Whitworth KW, Baird DD, Stene LC, et al. Fecundability among women with type 1 and type 2 diabetes in the Norwegian Mother and Child Cohort Study. Diabetologia. 2011;54(3):516-522. doi: 10.1007/s00125-010-2003-6

7. Eroglu A, Layman LC. Role of ART in imprinting disorders. Semin Reprod Med. 2012;30(2):92-104. doi: 10.1055/s-0032-1307417

8. Никонова Т.В., Витязева И.И., Пекарева Е.В., и др. Успешная беременность и роды у пациентки с сахарным диабетом 1-го типа и бесплодием при применении ЭКО и помповой инсулинотерапии (описание случая) // Проблемы репродукции. - 2015. - №3. C. 75-80. [Nikonova TV, Vityazeva II, Pekareva EV, et al. The successful in vitro fertilization in the patient with type 1 diabetes on insulin pump therapy (a case report). Problemy reproduktsii. 2015;(3):75-80. (In Russ.)] doi: 10.17116/repro 2015 21375-80

9. Dicker D, Ben-Rafael Z, Ashkenazi J, Feldberg D. In vitro fertilization and embryo transfer in well-controlled, insulin-dependent diabetics. Fertil Steril. 1992;58(2):430-432. doi: 10.1016/s0015-0282(16)55211-x

10. Oehninger S, Hofmann GE, Kreiner D, et al. Gonadotropin stimulation for in vitro fertilization and embryo transfer in insulin-dependent diabetics: Follicular response, oocyte quality, embryo development, and follicular environment. Int J Gynecol Obstet. 1990;33(4):379-379. doi: 10.1016/0020-7292(90)90533-q

11. Taylor CT. Review: Diabetes and assisted reproductive technology. Br J Diabetes Vasc Dis. 2016;2(4):247-253. doi: 10.1177/14746514020020040201

12. Bruttomesso D, Bonomo M, Costa S, et al. Type 1 diabetes control and pregnancy outcomes in women treated with continuous subcutaneous insulin infusion (CSII) or with insulin glargine and multiple daily injections of rapid-acting insulin analogues (glargine-MDI). Diabetes Metab. 2011;37(5):426-431. doi: 10.1016/j.diabet.2011.02.002

13. Тиселько А.В., Потин В.В., Боровик Н.В. Новейшие технологии в лечении беременных с сахарным диабетом 1 типа // Российский вестник акушера гинеколога. - 2017. - №5. - C. 35-40. [Tiselko AV, Borovik NV, Potin W. The latest technologies in the treatment of pregnant women with type 1 diabetes. Rossiyskiy vestnik akushera-ginekologa. 2017;(5):35-40. (In Russ.)] doi: 10.17116/rosakush201717535-40

14. Farrar D, Tuffnell DJ, West J. Continuous subcutaneous insulin infusion versus multiple daily injections of insulin for pregnant women with diabetes. Cochrane Database Syst Rev. 2007(3):CD005542. doi: 10.1002/14651858.CD005542.pub2

15. Handelsman Y, Mechanick J, Blonde L, et al. American Association of Clinical Endocrinologists Medical Guidelines for Clinical Practice for Developing a Diabetes Mellitus Comprehensive Care Plan: Executive Summary. Endocr Pract. 2011;17(2):287-302. doi: 10.4158/ep.17.2.287

16. Gardner DK, Schoolcraft WB. Culture and transfer of human blastocysts. Curr Opin Obstet Gynaecol. 1999;11(3):307-311. doi: 10.1097/00001703-199906000-00013

17. Григорян О.Р., Андреева Е.Н. Использование микронизированного натурального прогестерона в терапии нарушений менструального цикла у девушек с сахарным диабетом 1-го типа в сочетании с гипертриглицеридемией // Сахарный диабет. - 2008. T. 11. — №2. - C. 51-54. [Grigoryan OR, Andreeva EN. Ispol'zovanie mikronizirovannogo natural'nogo progesterona $v$ terapii narusheniy menstrual'nogo tsikla u devushek s sakharnym diabetom 1 tipa $v$ sochetanii s gipertriglitseridemiey. Diabetes mellitus. 2008;11(2):51-54. (In Russ.)] doi: 10.14341/2072-0351-5760

18. Miller E, Hare JW, Cloherty JP, et al. Elevated maternal hemoglobin A1C in early pregnancy and major congenital anomalies in infants of diabetic mothers. N Engl J Med. 1981;304(22):1331-1334. doi: 10.1056/NEJM198105283042204

19. Murphy HR, Rayman G, Lewis K, et al. Effectiveness of continuous glucose monitoring in pregnant women with diabetes: randomised clinical trial. BMJ. 2008;337:a1680. doi: 10.1136/bmj.a1680 
20. Дементьева Г.М. Оченка физического развития новорожденных: пособие для врачей. — M.; 2000. [Dement'eva GM. Otsenka fizicheskogo razvitiya novorozhdennykh: posobie dlya vrachey. Moscow; 2000. (In Russ.)]

21. Сахарный диабет и репродуктивная система женшины: руководство для врачей. / Под ред. Айламазяна Э.К. - М.: ГЭОТАР-Медиа, 2017. [Ajlamazjan JK, editor. Saharnyj diabet i reproduktivnaja sistema zhenshhiny: rukovodstvo dlja vrachej. Moscow: GEOTAR-Media; 2017. (In Russ.)]

22. Тиселько А.В., Боровик Н.В., Потин В.В. Эффективность различных режимов инсулинотерапии у больных с сахарным диабе- том 1-го типа во время беременности // Журнал акушерства и женских болезней. - 2013. - Т. 62. - №3. - C. 77-82. [Potin VV, Borovik NV, Tiselko AV. Efficacy of the different modes of insulin therapy in pregnant women with type 1 diabetes mellitus. Journal of Obstetrics and Women's Diseases. 2013;62(3):77-82. (In Russ.)] doi: 10.17816/JOWD62377-82

23. Battelino T, Conget I, Olsen B, et al. The use and efficacy of continuous glucose monitoring in type 1 diabetes treated with insulin pump therapy: a randomised controlled trial. Diabetologia. 2012;55(12):3155-3162. doi: 10.1007/s00125-012-2708-9

\section{ИНФОРМАЦИЯ ОБ АВТОРАХ [AUTHORS INFO]}

* Мишарина Елена Владимировна, к.М.н., врач акушер-гинеколог, С.н.с. [Elena V. Misharina, MD, PhD, senior research associate]; адрес: 199034, Санкт-Петербург, Менделеевская линия, д. 3 [address: Saint Petersburg, Mendeleyevskaya line 3, 199034 Russian Federation]; ORCID: http://orcid.org/0000-0002-0276-7112; eLibrary SPIN: 7350-5674; e-mail: mishellena@gmail.com

Тиселько Алена Викторовна, к.м.н., С.н.с. [Alena V. Tiselko, MD, PhD, senior research associate]; ORCID: http://orcid.org/0000-0002-2512-833X; eLibrary SPIN: 5644-9891; e-mail: alenadoc@mail.ru Мария Игоревна Ярмолинская, д.м.н., професcop [Maria I. Yarmolinskaya, MD, PhD, Professor]; ORCID: http://orcid.org/0000-0002-6551-4147; eLibrary SPIN: 3686-3605; e-mail: m.yarmolinskaya@gmail.com Коган Игорь Юрьевич, д.м.н., профессор, член-корреспондент РАH [lgor U. Kogan, MD, PhD, Professor]; ORCID: http://orcid.org/0000-0002-7351-6900; eLibrary SPIN: 4357-2017; e-mail: ikogan@mail.ru

Абашова Елена Ивановна; к.м.н., С.н.c. [Elena I. Abashova, MD, PhD, senior research associate]; ORCID: http://orcid.org/0000-0003-2399-3108; eLibrary SPIN: 2133-0310; e-mail: abashova@yandex.ru Боровик Наталья Викторовна, к.м.н., С.н.c. [Natalia V. Borovik, MD, PhD, senior research associate]; ORCID: http://orcid.org/0000-0003-0835-6741; eLibrary SPIN: 9010-7276; e-mail: borovik1970@yandex.ru

ЦИТИРОВАТЬ:

Мишарина Е.В., Тиселько А.В., Ярмолинская М.И., Коган И.Ю., Абашова Е.И., Боровик Н.И. Экстракорпоральное оплодотворение как метод лечения бесплодия у женщин с сахарным диабетом 1 типа // Caхарный диабет. 2018. — T. 21. — №5. — C. 425-430. doi: 10.14341/DM9573

\section{TO CITE THIS ARTICLE:}

Misharina EV, Tiselko AV, Yarmolinskaya MI, Kogan IY, Abashova El, Borovik NI. Vaccination and diabetes mellitus type 1 in children. Diabetes Mellitus. 2018;21(5):425-430. doi: 10.14341/DM9573 